\title{
$\mu$-Opioid Receptor-Mediated Inhibition of Intercalated Neurons and Effect on Synaptic Transmission to the Central Amygdala
}

\author{
Peter Blaesse, ${ }^{1 \star}$ Lena Goedecke, ${ }^{1 \star}$ Michaël Bazelot, ${ }^{2,3} \oplus^{\circledR}$ Marco Capogna, ${ }^{2}$ Hans-Christian Pape, ${ }^{1}$ and $\odot$ Kay Jüngling ${ }^{1}$ \\ ${ }^{1}$ Institute of Physiology I, University of Münster, D-48149 Münster, Germany, ${ }^{2}$ MRC Brain Network Dynamics Unit, Department of Pharmacology, \\ University of Oxford, Oxford, OX1 3TH, United Kingdom, and ${ }^{3} \mathrm{GW}$ Pharmaceuticals, School of Chemistry, Food and Nutritional Science and Pharmacy, \\ University of Reading, Whiteknights, Reading, RG6 6AP, United Kingdom
}

\begin{abstract}
The amygdala is a key region for the processing of information underlying fear, anxiety, and fear extinction. Within the local neuronal networks of the amygdala, a population of inhibitory, intercalated neurons (ITCs) modulates the flow of information among various nuclei of amygdala, including the basal nucleus (BA) and the centromedial nucleus (CeM) of the amygdala. These ITCs have been shown to be important during fear extinction and are target of a variety of neurotransmitters and neuropeptides. Here we provide evidence that the activation of $\mu$-opioid receptors (MORs) by the specific agonist DAMGO ([D-Ala2,N-Me-Phe4,Gly5-ol]-Enkephalin) hyperpolarizes medially located ITCs (mITCs) in acute brain slices of mice. Moreover, we use whole-cell patch-clamp recordings in combination with local electrical stimulation or glutamate uncaging to analyze the effect of MOR activation on local microcircuits. We show that the GABAergic transmission between mITCs and CeM neurons is attenuated by DAMGO, whereas the glutamatergic transmission on CeM neurons and mITCs is unaffected. Furthermore, MOR activation induced by theta burst stimulation in BA suppresses plastic changes of feedforward inhibitory transmission onto CeM neurons as revealed by the MOR antagonist CTAP d-Phe-Cys-Tyr-d-Trp-Arg-Thr-Pen-Thr-NH . $_{\text {. }}$ In summary, the mITCs constitute a target for the opioid system, and therefore, the activation of MOR in ITCs might play a central role in the modulation of the information processing between the basolateral complex of the amygdala and central nuclei of the amygdala.
\end{abstract}

Key words: central amygdala; MOR; opioids; plasticity

\section{Introduction}

Fear and extinction learning is heavily dependent on information processing in the neuronal networks within and between subnuclei of the amygdala (Ehrlich et al., 2009; Pape and Pare, 2010). Moreover, inhibitory networks in the amygdala were found to control the specificity of fear learning, thus preventing fear generalization (Lange et al., 2014), and are crucial for fear extinction (Paré et al., 2003; Likhtik et al., 2008; Ehrlich et al., 2009; Amano et al., 2010). Among the diverse inhibitory neurons of the amygdala, the subpopulation of intercalated neurons (ITCs) has

\footnotetext{
Received Jan. 15, 2015; revised Feb. 25, 2015; accepted March 12, 2015

Author contributions: P.B., L.G., M.B., M.C., H.-C.P., and K.J. designed research; P.B., L.G., M.B., and K.J. performed research; P.B., L.G., M.B., and K.J. analyzed data; P.B., L.G., M.C., H.-C.P., and K.J. wrote the paper.

This work was supported by German Research Foundation (Collaborative Research Centre TRR58) Grants TPA07 (K.J.) and TPA03 (H.-C.P.), a Cells in Motion International Max Planck Research School fellowship (L.G.), and the Wellcome Trust (M.C.). We thank E. Nass for expert technical assistance and P. Berenbrock and S. Kiesling for excellent animal care.

The authors declare no conflict of interest.

*P.B. and L.G. contributed equally to this work.

This article is freely available online through the J Neurosci Author Open Choice option.

Correspondence should be addressed to Dr. Kay Jüngling, Institute of Physiology I, Robert-Koch-Strasse 27a, University of Münster, D-48149 Münster, Germany. E-mail: Kay.Juengling@gmx.de.

DOI:10.1523/JNEUROSCI.0204-15.2015

Copyright $\odot 2015$ Blaesse, Goedecke et al.

This is an Open Access article distributed under the terms of the Creative Commons Attribution License Creative Commons Attribution 4.0 International, which permits unrestricted use, distribution and reproduction in any medium provided that the original work is properly attributed.
}

attracted much attention. The ITCs are located in a lateral cluster (IITC), predominantly modulating the information flow from cortical structures to the basolateral complex of the amygdala (BLA) and in a medial cluster (mITC), which appears as dorsal $[\operatorname{mITC}(\mathrm{d})]$ and ventral $[\mathrm{mITC}(\mathrm{v})]$ groups; the latter is also referred to as the main ITC nucleus (or IN cluster; Paré et al., 2003; Likhtik et al., 2008; Amano et al., 2010; Busti et al., 2011). The mITCs have been implicated in feedforward inhibition between the BLA/prefrontal cortex (PFC) and the centrolateral (CeL) and centromedial (CeM) nuclei of the central amygdala (CeA; Jüngling et al., 2008; Likhtik et al., 2008; Amano et al., 2010; Mańko et al., 2011). As an inhibitory gate between PFC, BLA, and the CeM, one of the main output nuclei of the amygdala, mITCs are important for fear extinction. For instance, interfering with mITC signaling attenuates extinction retrieval (Likhtik et al., 2008; Amano et al., 2010; Amir et al., 2011; for review, PalomaresCastillo et al., 2012).

ITCs are characterized by a high expression of the $\mu$-opioid receptor (MOR; Likhtik et al., 2008; Busti et al., 2011), a receptor for endogenous opioids (Bodnar, 2013). MORs can be activated by various endogenous ligands, such as enkephalins, endorphins, and endomorphins, with different potency (Zadina et al., 1999). Although MOR-coupled toxins have been used to eliminate mITCs from the amygdalar network and thereby interfere with fear extinction (Likhtik et al., 2008), the mechanisms of MOR 
activation in ITCs and its significance for amygdalar synaptic network activity have not yet been analyzed. The MORs are G-protein-coupled receptors, linked intracellularly to inhibitory G-proteins $\left(\mathrm{G}_{\mathrm{i}} / \mathrm{G}_{\mathrm{o}}\right)$, reducing the conductance of calcium channels and the activity of adenylyl cyclases and/or increasing the conductance of potassium channels (for review, see Law et al., 2000).

In the CeA, various actions of MOR agonists on neurons and synaptic transmission have been described in rats and mice (Zhu and Pan, 2004; Cabral et al., 2009). Behavioral experiments in rodents indicate that the $\mu$-opioid system is relevant for anxietyrelated behavior, stress coping, pain processing, and addiction (Tershner and Helmstetter, 2000; Bodnar, 2013; Lutz and Kieffer, 2013; Sandkühler and Lee, 2013). In humans, sustained pain leads to the release of endogenous opioids in the amygdala and other brain structures and activates MORs with antinociceptive consequences (Zubieta et al., 2001). This indicates that MOR activation is part of the antinociceptive system that is activated by stressful or fear-inducing environmental stimuli. Moreover, in chronic morphine-treated rats, extinction of cued conditioned fear has been shown to be impaired selectively (Gu et al., 2008).

We hypothesize that activation of MORs in mITCs reduces their activity via $G_{i} / G_{o}$ signaling and consequently modulates signal transfer between BA and CeM via mITCs. Whole-cell recordings from ITCs in acute slices were used to analyze postsynaptic effects in these neurons. Furthermore, we used either electrical microstimulation in the BA or local glutamate uncaging within the mITC cluster to characterize the functional consequence of MOR activation on synaptic transmission in microcircuits, in which mITCs display an inhibitory key structure.

\section{Materials and Methods}

The experimental design and animal handling was approved by the State Agency for Nature, Environment, and Consumer Protection of North Rhine-Westphalia (Reference 8.87-51.05.20.10.218) or was performed according to the Animals (Scientific Procedures) Act, 1986 (United Kingdom) and associated regulations.

Animals. C57BL/6J and transgenic GAD67-eGFP-expressing mice (Tamamaki et al., 2003) were kept in a temperature-controlled $\left(21^{\circ} \mathrm{C}\right)$ and humidity-controlled (50-60\% relative humidity) animal facility with access to food and water ad libitum and a $12 \mathrm{~h}$ light/dark cycle with lights on at 6:00 A.M. Food, water, and animal conditions were controlled on a daily basis.

Electrophysiology. Experimental procedures were done as described previously (Jüngling et al., 2008; Lange et al., 2012). In brief, 6- to 8-week-old male C57BL/6J or transgenic GAD67-eGFP-expressing mice were anesthetized with isoflurane (Abbott Laboratories) and decapitated. Some female mice were used to establish and test stimulation parameters. Coronal slices (300-350 $\mu \mathrm{m}$ thickness) containing the amygdala were prepared on a vibratome (VT1200S; Leica). Slices were placed in a submersion chamber at $30^{\circ} \mathrm{C}$ and were perfused with artificial CSF (aCSF) containing the following (in $\mathrm{mM}$ ): $120 \mathrm{NaCl}, 2.5 \mathrm{KCl}, 1.25$ $\mathrm{NaH}_{2} \mathrm{PO}_{4}, 2 \mathrm{MgSO}_{4}, 2 \mathrm{CaCl}_{2}, 22 \mathrm{NaHCO}_{3}$, and 20 glucose. The $\mathrm{pH}$ was set to 7.35 by gassing with carbogen. For recordings of synaptic responses, $1.5 \mathrm{mM} \mathrm{MgSO}_{4}$ and $2.5 \mathrm{mM} \mathrm{CaCl}_{2}$ were used in the extracellular solution.

Patch pipettes (2.5-4 $\mathrm{M} \Omega$ pipette resistance with a potassium gluconate-based intracellular solution were made of borosilicate glass (GC150T-10; Harvard Apparatus). The intracellular solution contained the following (in mM): $10 \mathrm{NaCl}, 105$ potassium gluconate, 20 potassium citrate, 10 HEPES, 3 BAPTA, $0.5 \mathrm{CaCl}_{2}, 1 \mathrm{MgCl}_{2}, 3 \mathrm{MgATP}, 0.5 \mathrm{NaGTP}$, and 15 phosphocreatin, $\mathrm{pH}$ adjusted to 7.25 . In some experiments, the intracellular solution contained the following (in $\mathrm{mM}$ ): 5 4-AP, 120 $\mathrm{CsMeSO}_{4}, 1$ EGTA, 10 HEPES 10, 20 tetraethylammonium-Cl, $2 \mathrm{MgCl}_{2}$, $0.5 \mathrm{CaCl}_{2}, 2 \mathrm{Na}-\mathrm{ATP}$, and $0.5 \mathrm{Na}-\mathrm{GTP}$.
TTX $(0.5 \mu \mathrm{M})$, DNQX $(10 \mu \mathrm{M})$, AP-5 $(50 \mu \mathrm{M})$, gabazine $(25 \mu \mathrm{M})$, and CGP55845 [(2S)-3-[(15)-1-(3,4-dichlorophenyl)ethyl] amino-2hydroxypropyl)(phenylmethyl)phosphinic acid $(2.5 \mu \mathrm{M})]$ were added when required. Drugs were purchased from Biozol Diagnostica. Electrophysiological data were acquired with an EPC10 double amplifier (HEKA) and analyzed offline with Clampfit10 software (Molecular Devices)

The number of generated action potentials (APs) was analyzed in response to a depolarizing current injection ( $40 \mathrm{pA}$ ) during current-clamp recordings. The input resistance was calculated as follows: $R_{\text {input }}=\Delta V / I$. $\Delta V$ was measured at the end of an injected hyperpolarizing current pulse $(I=-40 \mathrm{pA})$.

The MOR agonist [D-Ala ${ }^{2}$, NMe-Phe $\left.{ }^{4}, \quad G l y-\mathrm{ol}^{5}\right]$-enkephalin (DAMGO; R\&D Systems) was bath applied (3-3.5 $\mathrm{ml} / \mathrm{min}$ ) at a concentration of $250 \mathrm{~nm}$ or $2 \mu \mathrm{M}$. In initial experiments, neurons were recorded at the resting membrane potential. Later, the membrane potential during current-clamp recordings was set to $-60 \mathrm{mV}$ to achieve comparable driving forces independent of varying resting potentials. During maximal DAMGO effect, the membrane potential was set back manually to $-60 \mathrm{mV}$ with a direct current offset to either control the membrane resistance or perform a current-voltage relationship recording. The MOR-specific antagonist CTAP d-Phe-Cys-Tyr-d-Trp-Arg-Thr-PenThr- $\mathrm{NH}_{2}$ (R\&D Systems)] was used at a concentration of $500 \mathrm{~nm}$. To analyze substance-induced currents, the putative endogenous MOR ligands endomorphin-1 (Abcam) or DAMGO were bath applied (both at $250 \mathrm{~nm}$ ) for $5 \mathrm{~min}$ during voltage-clamp recordings at a holding potential of $-60 \mathrm{mV}$.

Depolarizing voltage-clamp ramps (from -120 to $-20 \mathrm{mV} ; 0.1 \mathrm{mV}$ / $\mathrm{ms}$ ) were repeated at least three times during baseline conditions (interval, $75 \mathrm{~s}$ ) and in the presence of $250 \mathrm{~nm}$ DAMGO. The extracellular solution contained TTX, DNQX, AP-5, gabazine, and CGP55845 to reduce network activity. The DAMGO-induced current was calculated by subtracting the ramp during baseline recordings from ramps recorded in the presence of DAMGO.

To evoke eEPSCs or eIPSCs, a tungsten bipolar stimulation electrode was placed in the basal nucleus of the amygdala (BA; see Fig. 2A). Brief $(50 \mu \mathrm{s})$ current pulses were applied with an interstimulus interval of $20 \mathrm{~s}$. Postsynaptic responses were recorded at $-65 \mathrm{mV}$ when isolated pharmacologically, at $-45 \mathrm{mV}$ when excitatory and inhibitory components were recorded, or at $-5 \mathrm{mV}$ to electrically isolate GABAergic eIPSCs. To induce long-term potentiation (LTP) of the disynaptic inhibitory component in CeM neurons, four sequences of theta burst stimuli (TBS) with an interstimulus interval of $25 \mathrm{~s}$ at a holding potential of $-5 \mathrm{mV}$ were applied. Each sequence consisted of four pulses at $100 \mathrm{~Hz}$ repeated 25 times at $2 \mathrm{~Hz}$ (Lange et al., 2012).

To normalize EPSCs or IPSCs, all recorded amplitudes of a given recording were divided by the mean amplitude of the baseline. Events smaller than two times the SD of the mean noise were considered as failures and excluded from analysis of the mean success amplitudes.

Glutamate uncaging. RuBi-glutamate (1 mM; Tocris Bioscience) diluted in aCSF was applied locally via a $250 \mu \mathrm{l}$ Hamilton syringe using an UltraMicroPump UMP3 (WPI). The syringe tip (100 $\mu \mathrm{m}$ diameter) was placed just above the slice surface. This configuration allows local application of high concentrations of caged compound to the area of interest but avoids recycling of small volumes of extracellular solution (Khirug et al., 2005). Uncaging parameters for local activation of the mITC(v) cluster were established by recording $\mathrm{mITC}(\mathrm{v})$ neurons in current-clamp mode. Near minimal responses in mITCs(v) were evoked when RuBiglutamate was photolyzed by scanning a circular region of interest (ROI; $\sim 1 \mu \mathrm{m}$ diameter) placed on the recorded cell with $22.5 \pm 3 \%$ of the maximal intensity of a $473 \mathrm{~nm}$ laser $(\sim 40 \mathrm{~ms}$ scan time). The same uncaging settings were used when CeM neurons were recorded in voltage-clamp mode at a holding potential of $-5 \mathrm{mV}$ to isolate GABAergic responses and when the uncaging ROI was placed within the mITC(v) cluster. After GABAergic IPSCs in CeM neurons were recorded under baseline conditions, DAMGO was bath applied at a concentration of $250 \mathrm{~nm}$ for $5 \mathrm{~min}$. Events smaller than two times the SD of the mean noise were considered as failures. Success amplitudes and failure rates were analyzed during baseline, in the presence of DAMGO, and after washout. 


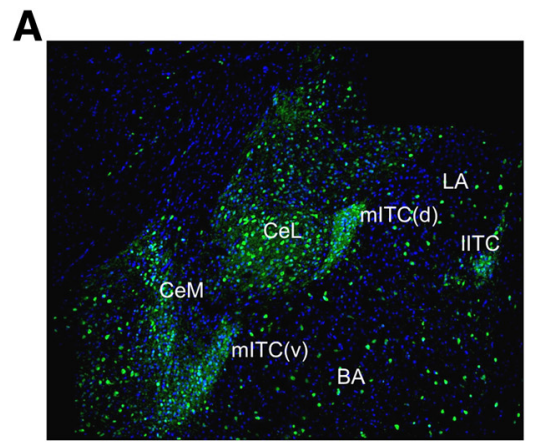

B

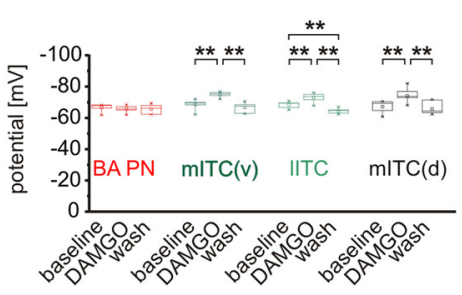

C
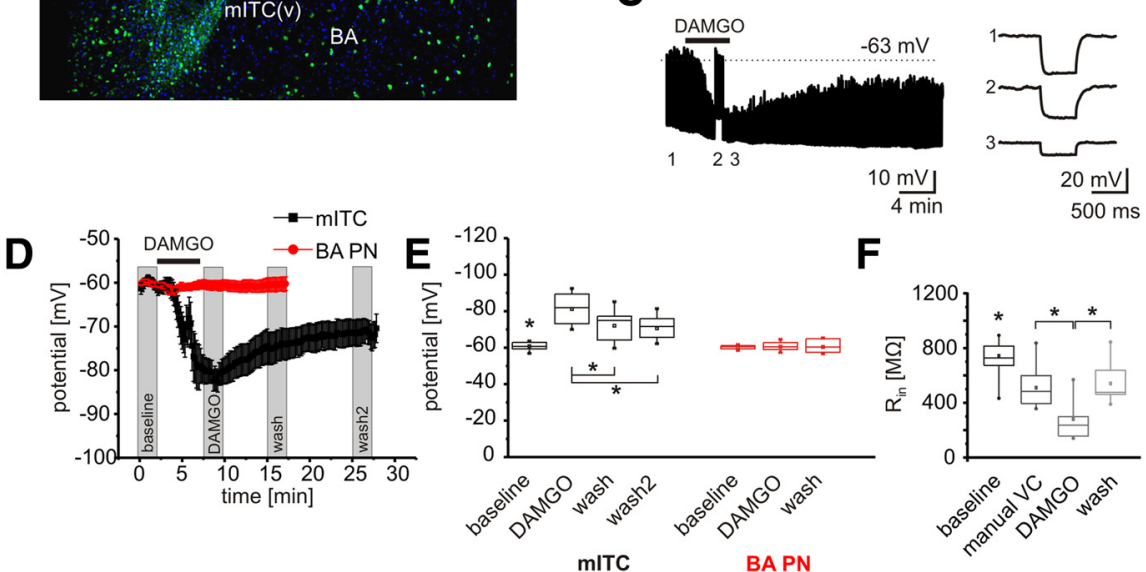

$\mathbf{F}$
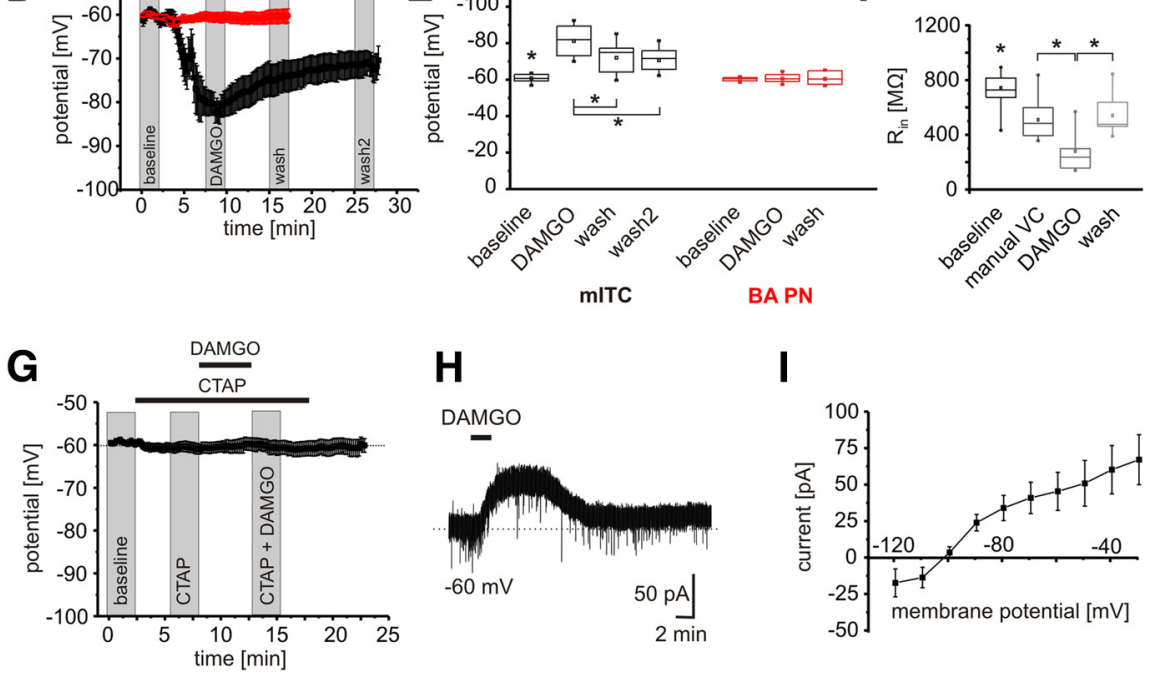

Figure 1. Activation of MORs hyperpolarizes mITCs of the amygdala. A, Localization of the mITC(d)s and mITC(v)s in the amygdala of a transgenic GAD67- eGFP mouse. Interneurons were identified by their green fluorescence. Neuronal nuclei are stained with DAPI (blue). Current-clamp recordings were done in both $\mathrm{mITC}(\mathrm{d})$ s and $\mathrm{mITC}(\mathrm{v}) \mathrm{s}$. B, Quantification of the DAMGOdependent change of the membrane potential in BA PNs $(n=6 / 5), \operatorname{mITC}(v)(n=10 / 8)$, IITC $(n=7 / 4)$, and $\operatorname{mITC}(\mathrm{d})(n=8 / 8)$ recorded in current-clamp mode at resting membrane potential (2 $\mu \mathrm{M}$ DAMGO). C, Example recording of an mITC in current-clamp mode showing a significant hyperpolarization in response to DAMGO $(250 \mathrm{nM})$. Hyperpolarizing responses to a $-40 \mathrm{pA}$ current injection are presented enlarged for baseline, maximal DAMGO effect, and at $-63 \mathrm{mV}$ in the presence of DAMGO (manual VC). The input resistance of the recorded neuron was calculated during baseline conditions (1), in the presence of DAMGO while applying manual VC (2), and during maximal hyperpolarization under DAMGO (3). Manual VC was used to isolate substance-induced effects from hyperpolarization-induced effects on the input resistance. $\boldsymbol{D}$, Time course of the membrane potential recorded in $\mathrm{mITCs}$ and BA PNs $(n=6 / 5)$. Gray boxes (covering 2.5 min of recording) indicate the time points used for analysis of the membrane potential during the different recording conditions (baseline, DAMGO [mITC(v): $n=11 / 5]$, wash [mITC(v): $n=10 / 5]$, wash2 [mITC(v): $n=$ 8/4]). $\boldsymbol{E}$, Quantification of the mean membrane potential for mITCs and BA neurons at the respective time points shown in $\boldsymbol{D} . \boldsymbol{F}$, Quantification of the input resistance in the absence and presence of DAMGO $(n=11 / 5)$ and after wash $(n=10 / 5)$ in $\mathrm{mITC}$. $\boldsymbol{G}$, Time course of the membrane potential during baseline conditions, in the presence of CTAP, and in the presence of CTAP and DAMGO ( $n=8 / 2)$. Gray boxes indicate the time points used for analysis of the membrane potential during the different recording conditions (baseline, (TAP, and (TAP + DAMGO). $\boldsymbol{H}$, Example recording of an mITC in voltage-clamp mode at a holding potential of $-60 \mathrm{mV}$. Bath application of DAMGO induced an outward-directed current. I, Quantification of the mean DAMG0-induced current calculated by ramp $P_{D A M G O}-$ ramp $_{\text {baseline. }}$. The apparent reversal potential of the DAMG0-induced current was at $-103 \pm$ $1.7 \mathrm{mV}(n=6 / 3)$.

Statistics. All datasets were tested for significant outliers using the Grubbs' test (significance level, $p<0.05$ ). Data were tested for significance using a one-way ANOVA, followed by a Bonferroni's post hoc test. In some cases, an unpaired Student's $t$ test was used (within-group comparisons). To compare the time course of normalized amplitudes, the Kolmogorov-Smirnov test was used. Statistical tests were performed with OriginPro 7.5G (OriginLab). Data are represented as box plots, in which the box represents the first $(25 \%)$ and third $(75 \%)$ quartiles, the band represents the median, and the whiskers represent the percentiles $5 \%$ and $95 \%$. The square within the box represents the mean. The num- ber of experiments is given as number of cells/ number of animals throughout. The level of significance is given by ${ }^{*} p<0.05$ and ${ }^{* *} p<$ 0.01 .

\section{Results}

\section{Inhibition of ITCs during MOR} activation by DAMGO

The ITCs are characterized by a high expression level of MORs (Amir et al., 2011; Busti et al., 2011), but direct electrophysiological evidence for MOR action on ITCs is missing. Recordings on ITCs and $\mathrm{BA}$ principal neurons (PNs) were done either in C57BL/6J mice or transgenic GAD67-eGFP-expressing mice (Fig. 1A). ITCs were readily identified by their morphology, location, and electrophysiological properties (Jüngling et al., 2008; Busti et al., 2011) and/or expression of eGFP in transgenic animals (Fig. 1A). In a first set of experiments, whole-cell recordings of ITCs and BA PNs were performed in current-clamp mode. In all recorded ITCs $(n=25)$, application of $2 \mu \mathrm{M}$ DAMGO induced a transient hyperpolarization from resting membrane potential, whereas it had no effect on BA PNs (Fig. 1B). In BA PNs, the mean membrane resting potential was at $-67 \pm 1 \mathrm{mV}$ during baseline, $-66 \pm 1$ $\mathrm{mV}$ in the presence of $2 \mu \mathrm{m}$ DAMGO, and $-66 \pm 1 \mathrm{mV}$ after wash (one-way ANOVA: $\left.F_{(2,15)}=0.3, p=0.75 ; n=6 / 5\right)$. In contrast, DAMGO hyperpolarized the $\operatorname{mITC}(\mathrm{v})$ from $-69 \pm 0.9$ to $-75 \pm 0.5$ $\mathrm{mV}$, and the membrane potential returned to $-67 \pm 1.1 \mathrm{mV}$ after wash (oneway ANOVA: $F_{(2,24)}=30.2, p=0.000001$; post hoc test: baseline vs DAMGO, $p=$ 3.9595E-6; DAMGO vs wash, $p=$ $1.2576 \mathrm{E}-6 ; n=10 / 8)$. The IITCs were hyperpolarized from $-68 \pm 0.8$ to $-73 \pm 1$ $\mathrm{mV}$ in the presence of DAMGO, and they reversed to $-64 \pm 0.7 \mathrm{mV}$ after wash (one-way ANOVA: $F_{(2,17)}=25.4, p=$ 0.00001; post hoc test: baseline vs DAMGO, $p=0.0034$; DAMGO vs wash, $p=3.0208 \mathrm{E}-5$; baseline vs wash, $p=$ $0.0024 ; n=7 / 4)$. The mITC(d) hyperpolarized from $-67 \pm 1.3$ to $-74 \pm 1.5 \mathrm{mV}$ in the presence of DAMGO and reversed to $-66 \pm 1.3 \mathrm{mV}$ after wash (one-way ANOVA: $F_{(2,21)}=10.9, p=0.0006$; post hoc test: baseline vs DAMGO, $p=0.003$; DAMGO vs wash, $p=8.0203 \mathrm{E}-4 ; n=8 / 8$ ). In summary, neurons from all tested ITC clusters hyperpolarized in response to MOR activation by DAMGO, but DAMGO failed to induce a change in the membrane potential of BA PNs.

In the following, the effects of DAMGO on mITCs were analyzed in detail. To differentiate between direct postsynaptic DAMGO-dependent effects on mITCs or indirect network effects, current-clamp recordings in the presence or absence of 
TTX were performed to suppress AP-dependent network activity. In contrast to the previous experiments, the membrane potential during current-clamp recordings was set to $-60 \mathrm{mV}$ via manual voltage clamp (manual VC) to achieve comparable driving forces for all mITCs independent of their resting membrane potential. DAMGO was bath applied at a concentration of 250 nM. DAMGO induced a prominent hyperpolarization in mITCs in the presence of TTX (baseline, $-60 \pm 0.8 \mathrm{mV}$; DAMGO, $-81 \pm 2.2 \mathrm{mV}$; $t$ test: $t=7.86, \mathrm{df}=6, p=2.2445 \mathrm{E}-4 ; n=7 / 2)$ or in the absence of TTX (baseline, $-62 \pm 0.7 \mathrm{mV}$; DAMGO, $-82 \pm 5 \mathrm{mV} ; t$ test: $t=4.24, \mathrm{df}=3, p=0.024 ; n=4 / 3)$. The persistence of the DAMGO-induced hyperpolarization indicates that the observed changes of the membrane potential are attributable to a direct postsynaptic effect and less likely via indirect network effects. Because the DAMGO-induced effect did not differ in the presence or absence of TTX (DAMGO with TTX vs DAMGO without TTX: one-way ANOVA: $F_{(1,9)}=0.079, p=$ 0.78 ), the data were pooled (Fig. $1 E$ ). In the presence of DAMGO, mITCs hyperpolarized from $-61 \pm 0.6 \mathrm{mV}(n=11 / 5)$ to $-81 \pm$ $2.2 \mathrm{mV}(n=11 / 5)$, and the effect declined to $-72 \pm 2.8 \mathrm{mV}(n=$ $10 / 5)$ after $5 \mathrm{~min}$ (wash) and to $-71 \pm 2.3(n=8 / 4)$ after $15 \mathrm{~min}$ (wash2; Fig. 1D). The observed effect of the DAMGO-induced hyperpolarization was statistically significant (one-way ANOVA: $F_{(3,36)}=17.16, p=0.00001$; post hoc test: baseline vs DAMGO, $p=2.9794 \mathrm{E}-8$; baseline vs wash, $p=5.4906 \mathrm{E}-4$; baseline vs wash2, $p=2.4745 \mathrm{E}-4$; DAMGO vs wash2, $p=0.0056$; Fig. $1 E$ ). In contrast to mITCs, BA PNs did not show any significant change of the membrane potential in the presence of DAMGO. The membrane potential during baseline conditions was at $-60 \pm 0.5$ $\mathrm{mV}(n=6 / 5)$, at $-61 \pm 1 \mathrm{mV}$ in the presence of DAMGO, and at $-60 \pm 1.6 \mathrm{mV}$ after wash (Fig. $1 D, E$ ). The membrane potential during maximal hyperpolarization in mITCs was significantly different from BA PNs (ITC DAMGO vs BA PN DAMGO: oneway ANOVA: $F_{(1,15)}=41.9, p=0.00001$; post hoc test: $p=$ 1.0502E-5).

In mITCs, the MOR-induced membrane hyperpolarization was accompanied by a reduction in apparent membrane input resistance. To prevent a contamination of the calculated resistance by voltage-dependent changes of the conductance during maximal hyperpolarization, manual VC was used to set the membrane potential back to baseline values (Fig. 1C). The initial membrane resistance of $735 \pm 39 \mathrm{M} \Omega(n=11 / 5)$ was reduced to $286 \pm 47 \mathrm{M} \Omega$ during the maximal DAMGO effect (without manual VC) and was still different from baseline when manual VC was applied $(510 \pm 42 \mathrm{M} \Omega$ ). The membrane resistance partially recovered after wash to $549 \pm 45 \mathrm{M} \Omega(n=10 / 5)$. The changes of the membrane resistance were significant (one-way ANOVA: $F_{(3,39)}=18.46, p=0.00001$; post hoc test: baseline vs DAMGO manual VC, $p=8.6302 \mathrm{E}-4$; baseline vs maximal DAMGO, $p=4.3481 \mathrm{E}-7$; maximal DAMGO vs wash, $p=$ 7.2545E-4; Fig. $1 F)$.

To verify specificity of MOR activation in mITCs, DAMGO was applied in the presence of the MOR antagonist CTAP (Fig. $1 G)$. Neither CTAP alone nor DAMGO in the presence of CTAP induced a significant change in membrane potential (baseline, $-59.4 \pm 1.6 \mathrm{mV}$; CTAP, $-60.6 \pm 1.2 \mathrm{mV}$; CTAP + DAMGO, $-60 \pm 4 \mathrm{mV} ; n=8 / 2$ ). During current-clamp recordings at a membrane potential of $-60 \mathrm{mV}$, DAMGO reduced the number of generated APs in response to depolarizing current injections $(40 \mathrm{pA})$ from $10.3 \pm 2.1$ APs under baseline conditions to $5 \pm 1.3$ $\operatorname{APs}(p=0.037 ; n=9 / 3)$.

In summary, activation of MORs in mITCs induces a prominent, CTAP-sensitive membrane hyperpolarization that is ac- companied by a reduction in membrane input resistance. The observed effects were independent of synaptic network activity, because the recordings were performed in the presence of TTX and glutamate and $\mathrm{GABA}_{\mathrm{A} / \mathrm{B}}$ receptor antagonists.

To characterize the ionic mechanisms underlying the DAMGO-induced hyperpolarization, voltage-clamp recordings of mITCs were performed at a holding potential of $-60 \mathrm{mV}$. DAMGO (250 nM) induced a significant outward current (baseline, $1.9 \pm 1.09 \mathrm{pA}$; DAMGO, $34.5 \pm 4.6 \mathrm{pA}$; wash, $15.3 \pm 3.7 \mathrm{pA}$; $n=6 / 2$; one-way ANOVA: $F_{(2,15)}=22.2, p=0.00003$; post hoc test: baseline vs DAMGO, $p=4.5099 \mathrm{E}-5$; baseline vs wash, $p=$ 0.0059; DAMGO vs wash, $p=0.0087$; Fig. $1 H$ ).

Voltage-clamp ramp recordings during baseline and in the presence of DAMGO revealed that the DAMGO-induced outward current displays a reversal potential $\left(E_{\mathrm{rev}}\right)$ of $-103 \pm 1.7 \mathrm{mV}(n=6 / 3$; Fig. $1 I)$. The $E_{\text {rev }}$ indicates that the DAMGO-induced current in mITCs is mainly carried by potassium ions.

Similar to the DAMGO-induced currents, the potential endogenous MOR agonist endomorphin-1 induced a significant outward current in mITCs (baseline, $-0.2 \pm 0.4 \mathrm{pA}$; endomorphin-1, $22 \pm 2.03 \mathrm{pA}$; wash, $7 \pm 1.3 \mathrm{pA} ; n=4 / 2$; oneway ANOVA: $F_{(2,9)}=78.8, p=1.98 \mathrm{E}-6$; post hoc test: baseline vs endomorphin-1, $p=0.001$; endomorphin-1 vs wash, $p=0.011$; baseline vs wash, $p=0.016$; data not shown).

\section{MOR activation reduces inhibitory but not excitatory components of an intra-amygdalar network}

Neurons of the mITC have been shown to be active during fear extinction (Busti et al., 2011) and control the signal transfer from the BA to the CeA (Royer et al., 1999; Likhtik et al., 2008; Amano et al., 2010). Our next experiments focused on the mITC(v) neurons because these neurons are ideally situated to control neuronal targets in the CeM. Based on our previous findings, we hypothesized that activation of MORs in the mITC(v) alters the signal transfer from BA to CeM.

In a first set of experiments, local, electrical stimulation in BA was used, and synaptic responses were recorded in CeM neurons at a holding potential of $-45 \mathrm{mV}$. Figure $2 \mathrm{~A}$ depicts putative synaptic connections between the BA and CeM that possibly contribute to recorded excitatory and inhibitory synaptic components. The analyzed responses were composed of a monosynaptic excitatory (eEPSC) and disynaptic inhibitory (eIPSC) component. Both eEPSCs and eIPSCs were sensitive to the glutamate receptor antagonist DNQX, indicating that the eIPSC component is driven by excitatory synapses on inhibitory neurons, projecting onto CeM neurons (Fig. 2B). Application of DAMGO $(250 \mathrm{nM})$ induced a significant reduction of the eIPSC amplitude $[0.53 \pm 0.08(n=9 / 4)$ in the presence of DAMGO and $0.72 \pm$ 0.11 when normalized to baseline; ANOVA: $F_{(2,24)}=9.21, p=$ 0.001 ; post hoc test: baseline vs DAMGO, $p=3.488 \mathrm{E}-5$; Fig. $2 C-$ $E]$. Concomitantly, the failure rate of the evoked responses tended to increase in the presence of DAMGO (baseline, $8.5 \pm$ 5.8\%; DAMGO, $35.4 \pm 10.8 \%$; wash, $24.4 \pm 10.2$; Fig. $2 F)$, although the effect was not significant (one-way ANOVA: $F_{(2,24)}=$ $2.15, p=0.138$ ). No effects of MOR activation on eEPSCs in CeM neurons were detected. Amplitudes of eEPSC were at $0.95 \pm 0.07$ in the presence of DAMGO and at $0.87 \pm 0.1$ after wash (relative to the baseline condition; $n=9 / 4$; Fig. $2 C, G$ ). In addition to the observed synaptic effects, in a subset of CeM neurons, a DAMGO-induced outward current was detected that was most likely mediated by activation of MORs located on CeM neurons. Indeed, in 10 recorded CeM neurons, DAMGO elicited a mean outward current of $17.2 \pm 10.4 \mathrm{pA}$ that declined slightly to $12.3 \pm$ 

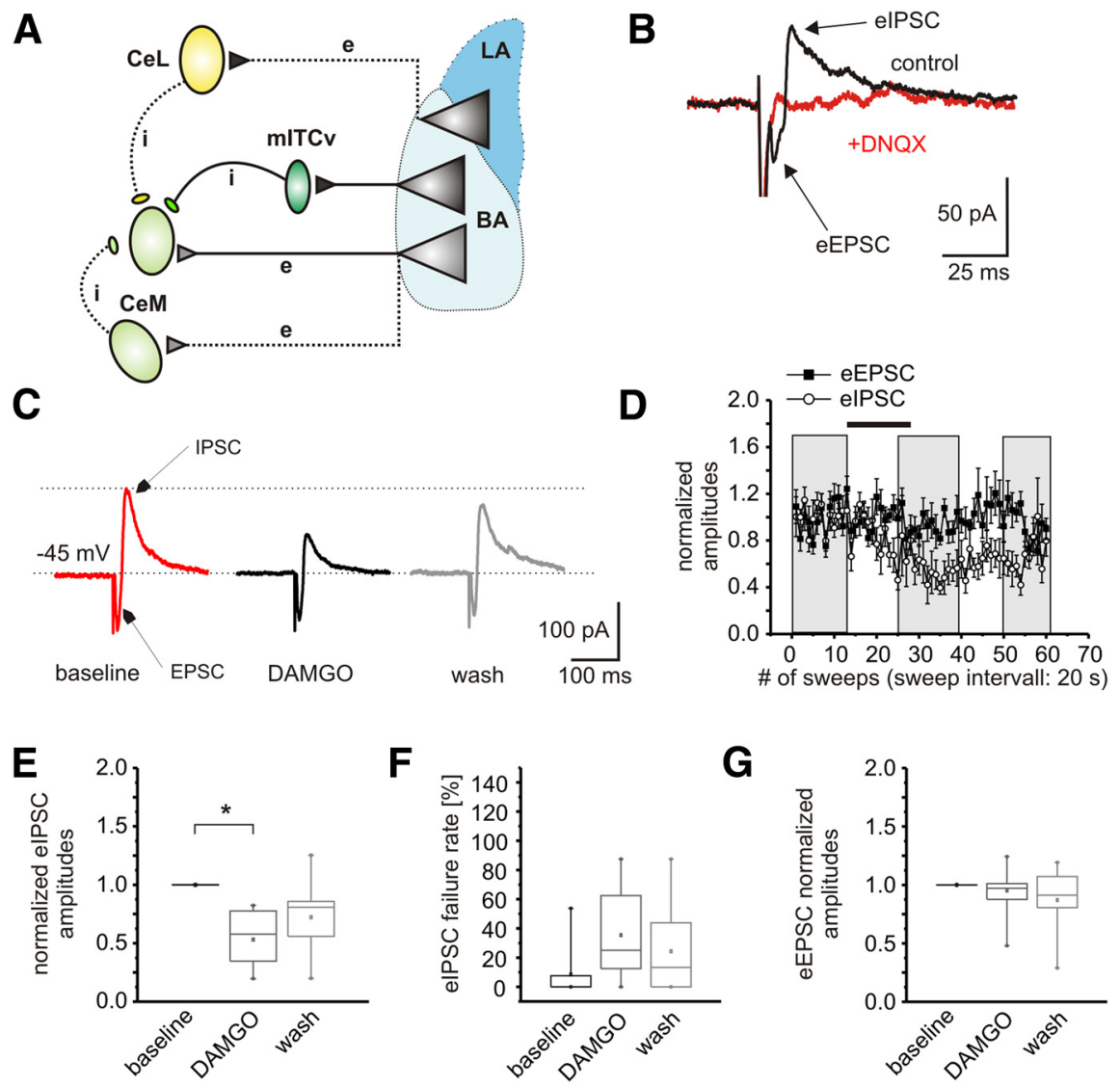

Figure 2. Disynaptic GABAergic responses recorded in CeM neurons are attenuated by DAMGO. $A$, Scheme of possible synaptic connections (excitatory, e; inhibitory, i) contributing to disynaptic responses recorded in CeM neurons after BA stimulation. $B, C$, Example recording of a CeM neuron in voltage-clamp mode at a holding potential of $-45 \mathrm{mV}$. BA stimulation resulted in a biphasic response, composed of a monosynaptic glutamatergic (EPSC) and a disynaptic GABAergic (IPSC) component. Application of DNQX abolished disynaptic GABAergic components. Application of DAMGO reduced the GABAergic component of the evoked response. $\boldsymbol{D}$, Time course of the mean normalized amplitudes of evoked EPSCs and IPSCs. Black bar indicates the application of DAMGO. Gray boxes represent time points used for analysis of the evoked responses during the different recording conditions (baseline, DAMGO, and washout; $n=9 / 4)$. Mean amplitudes and failure rates were calculated from evoked responses within these time segments. $\boldsymbol{E}_{i}$ $\boldsymbol{F}$, Quantification of the normalized success amplitude $(\boldsymbol{E})$ and failure rate $(\boldsymbol{F})$ of evoked IPSCs recorded from CeM neurons $(n=$ 9/4). G, Quantification of the normalized success amplitude of evoked EPSCS ( $n=9 / 4)$.

$10.4 \mathrm{pA}$ after wash $(n=10 / 4)$. Of the 10 recorded neurons, four did show a large outward current of $45.8 \pm 18.3 \mathrm{pA}$, indicating that a subpopulation of CeM neurons expresses MORs. Overall, these findings provide evidence that (1) the inhibitory synaptic component recorded in CeM neurons after BA stimulation is attenuated by MOR activation and (2) MORs are located in presynaptic inhibitory neurons and in addition at postsynaptic sites in a subset of CeM neurons.

Recordings of BA-induced disynaptic IPSCs in CeM neurons indicated an effect of MOR activation in mITCs on BA-to-CeM transmission; however, the interpretation of the results is hampered by the temporal overlap of EPSCs and IPSCs. Therefore, in the next approach, $\mathrm{GABA}_{\mathrm{A}}$ and $\mathrm{GABA}_{\mathrm{B}}$ receptor-mediated responses were blocked by gabazine $(25 \mu \mathrm{M})$ and CGP55845 (2.5 $\mu \mathrm{M})$, respectively, and pharmacologically isolated eEPSCs were recorded at a holding potential of $-65 \mathrm{mV}$ in $\mathrm{mITC}(\mathrm{v}) \mathrm{s}$ and $\mathrm{CeM}$ neurons (Fig. 3). Electrical stimulation within the BA evoked monosynaptic eEPSCs in $\operatorname{mITC}(v)$ neurons with a mean amplitude of $-47 \pm 11 \mathrm{pA}(n=6 / 4$; Fig. $3 B)$. DAMGO had no significant effect on normalized EPSC amplitudes (DAMGO, $0.86 \pm 0.07$; wash, $0.84 \pm 0.14$; Fig. $3 C, D$ ). Furthermore, monosynaptic eEPSCs in CeM neurons (Fig. $3 F$ ) did not show alterations during application of DAMGO (Fig. $3 F, G$ ). The mean normalized amplitudes were at $0.98 \pm 0.07$ in the presence of DAMGO and at $1.09 \pm 0.09$ after wash $(n=9 / 3$; Fig. $3 H)$. Also under these recording conditions, a transient outward current in CeM neurons was detectable during DAMGO application and subsequent MOR activation in CeM neurons with a mean amplitude of $17.2 \pm 5.8$ $\mathrm{pA}$, confirming the additional presence of postsynaptic MORs in a subset of CeM neurons. These data indicate that BA neurons are not directly modulated by DAMGO and that the observed reduction of the disynaptic eIPSC in CeM neurons in the presence of DAMGO is not contaminated by changes in excitatory drive of $\operatorname{mITC}(\mathrm{v})$ neurons but rather reflect a response of the mediating GABAergic neurons.

Neuroanatomical and electrophysiological data indicate that a subset of mITCs axons terminate on neurons within the CeM. To detect a possible direct synaptic connection between mITCs and CeM neurons and to analyze the effects of MOR activation onto these connections, local glutamate uncaging within the mITC(v) cluster was used (Fig. 4). The rational was that APs are evoked by local glutamate receptor activation within the mITC cluster. If synaptic connections between mITCs and CeM neurons exist, APs within mITCs will result in IPSCs that can be recorded in CeM neurons. Restricted ROIs were scanned with a mean laser intensity of $22 \pm 3 \%$ of the maximal laser intensity and mean scan duration of $40.5 \pm 3.6 \mathrm{~ms}$ (depending on ROI size). In control experiments, local glutamate uncaging reliably evoked APs in recorded mITCs (Fig. 4A). The probability to evoke APs declined with increasing distance from visible neuronal structures (Fig. $4 A, B$ ). These data indicate that glutamate uncaging allows a locally restricted stimulation of mITC(v) neurons within the cluster without substantial crossactivation of surrounding tissue. During recordings of CeM neurons, GABAergic eIPSCs were isolated using a cesium-based intracellular solution and a holding potential of $-5 \mathrm{mV}$. Glutamate uncaging in the mITC(v) cluster evoked IPSCs (Fig. 4D) with a mean amplitude of $16.6 \pm 3.9 \mathrm{pA}$ and a mean failure rate of $24 \pm 5 \%(n=9 / 7$; Fig. $4 D-F)$. IPSCs evoked by glutamate uncaging had a mean latency of $29 \pm 1.6 \mathrm{~ms}$, and the eIPSC always occurred within the laser scan time. It should be noted that, in contrast to electrical stimulation, the latency after glutamate uncaging depends not only on the time course of AP generation and synaptic transmission but also on the laser scan time and the local rise of the glutamate concentration. Moreover, the distance of the ROI to the site of AP generation influences greatly the latency to AP onset (Fig. 4A). To analyze the effect of DAMGO, the normalized eIPSC amplitude and the failure rate were calculated during baseline, in the presence of DAMGO, and after wash. Bath application of DAMGO had no significant effect on eIPSC amplitude 
(one-way ANOVA: $F_{(2,22)}=2.71, p=$ $0.09 ; n=9 / 7$; Fig. $4 E, F)$. In contrast, DAMGO transiently increased the failure rate of the eIPSCs from $24 \pm 5$ to $61 \pm$ $11 \%$, returning to $48 \pm 8 \%$ after wash (one-way ANOVA: $F_{(2,24)}=4.825, p=$ 0.017; post hoc test: baseline vs DAMGO, $p=0.0018 ; n=9 / 7$; Fig. $4 E, F)$. These data indicate that DAMGO decreases the GABA release probability from mITCs onto CeM neurons by either preventing AP generation attributable to an induced hyperpolarization or by directly affecting the presynaptic release machinery but does not grossly alter the amplitude by modulating postsynaptic GABA receptors.

\section{MOR blockage permits disynaptic GABAergic plasticity in CeM neurons} Because MOR activation negatively modulated inhibitory inputs onto CeM neurons, we hypothesized that endogenous MOR ligands inhibit disynaptic plasticity in CeM. Whole-cell recordings of CeM neurons were performed at a holding potential of $-5 \mathrm{mV}$ using a cesiumbased intracellular solution. Disynaptic responses were evoked by applying brief current pulses within the BA (Fig. 5A). TBS was used in the presence (CTAP) or absence (control) of the MOR-specific antagonist CTAP. All recordings were done in the absence of glutamate receptor antagonists, and CGP55848 was added to the aCSF to prevent a contribution of $\mathrm{GABA}_{\mathrm{B}}$ receptors, because a possible contribution of presynaptically located $\mathrm{GABA}_{\mathrm{B}}$ receptors might suppress transmitter release during stimulation and thus hinder a differentiation between $\mathrm{GABA}_{\mathrm{B}}$ receptor- and MOR-dependent effects. Only success amplitudes were included to calculate mean absolute or normalized amplitudes. During baseline stimulation, there was no significant difference between the control and CTAP groups. The mean amplitude of the control group was $51.3 \pm 8.7 \mathrm{pA}(n=13 / 8)$ and $42.9 \pm 6.4$ pA $(n=16 / 8)$ in the CTAP group (Fig. $5 B)$. The normalized amplitudes in the control group were at $0.99 \pm 0.09$ during the first $5 \mathrm{~min}$ after TBS (postTBS I) and at $0.65 \pm 0.07$ during the last $5 \mathrm{~min}$ after TBS (postTBS II; Fig. 5B-D). The decline of the normalized amplitudes after TBS was significant (one-way ANOVA: $F_{(2,36)}=$ 9.44, $p=0.0005$; post hoc test: baseline vs postTBS II, $p=5.3947 \mathrm{E}-5$; postTBS I vs postTBS II, $p=0.0057$; Fig. $5 D$ ). In contrast, TBS in the presence of CTAP led to a significant increase of the normalized amplitudes at postTBS I to $1.52 \pm 0.19$ and to $1.12 \pm 0.12$ postTBS II (one-way ANOVA: $F_{(2,43)}=4.31$; post hoc test: baseline vs

E
B
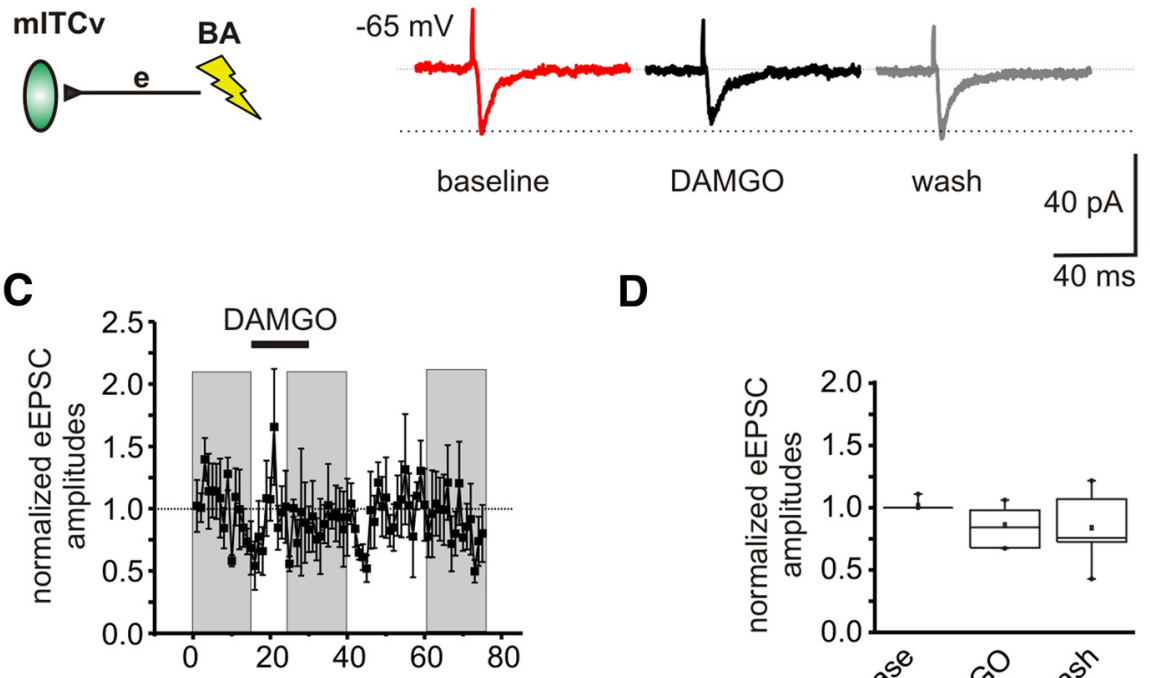

\# of sweeps (sweep intervall: $20 \mathrm{~s}$ )

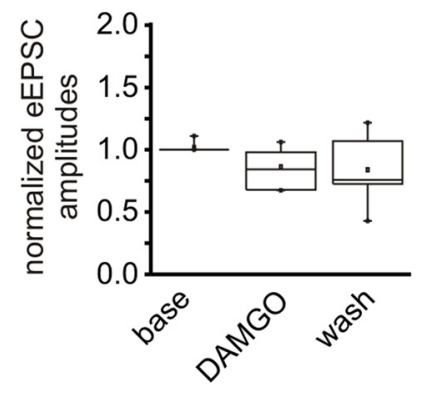

$\mathbf{F}$
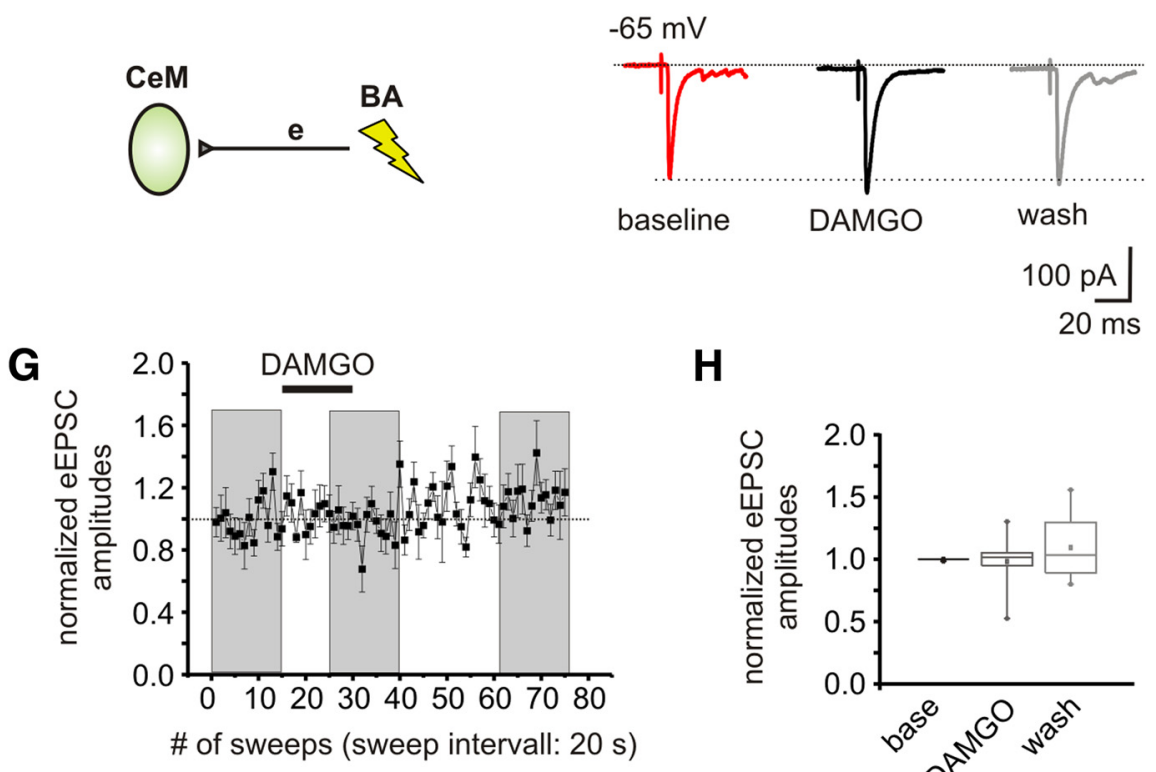

H

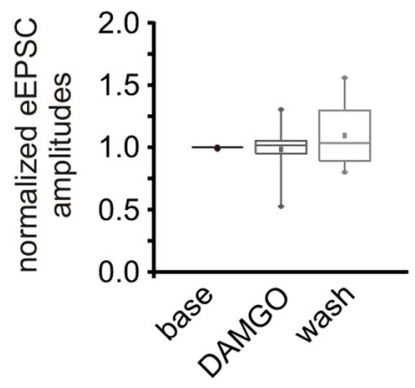

Figure 3. Monosynaptic eEPSCs on mITC(v)s and CeM neurons are unaffected by DAMGO. $\boldsymbol{A}$, Schematic presentation of the stimulation site within the BA (to evoke EPSCs) and of the recording site within the mITC(v) cluster. e, Excitatory. $B$, Example recording of an mITC(v) neuron in voltage-clamp mode at a holding potential of $-65 \mathrm{mV}$ in the presence of $G_{A B A_{A}}$ and $G_{A B A_{B}}$ receptor antagonists. Electrically evoked EPSCs recorded from mITCs seemed to be unaffected by DAMGO. C, Time course of the mean normalized amplitudes of eEPSCs during baseline conditions, in the presence of DAMGO, and after washout. Gray boxes represent time points used for analysis of the evoked responses during the different recording conditions (baseline, DAMG0, and washout). Mean amplitudes were calculated from evoked responses within these time segments ( $n=6 / 4)$. D , Quantification of the normalized eEPSC amplitudes $(n=6 / 4)$. $E$, Schematic presentation of the stimulation site within the BA (to evoke EPSCs) and of the recording site within the CeM. $\boldsymbol{F}$, Example recording of a CeM neuron in voltage-clamp mode at a holding potential of $-65 \mathrm{mV}$ in the presence of $G A B A_{A}$ and $G A B A_{B}$ receptor antagonists. Electrically evoked EPSCs recorded from CeM neurons seemed to be unaffected by DAMGO. $\boldsymbol{G}, \boldsymbol{H}$, Time course $(\boldsymbol{G})$ and quantification $(\boldsymbol{H})$ of the mean normalized amplitudes of eEPSCs during baseline conditions, in the presence ofDAMG0, and after washout. Gray boxes represent time points used for analysis of the evoked responses during the different recording conditions (baseline, DAMG0, and washout). Mean amplitudes were calculated from evoked responses within these time segments $(n=9 / 3)$.

postTBS I, $p=0.014$; Fig. $5 C, D)$. The time course of the normalized amplitudes recorded under control conditions or in the presence of CTAP was significantly different (KolmogorovSmirnov test: $p=2.1427 \mathrm{e}-13$; difference, 0.29005; Fig. 5C). The 

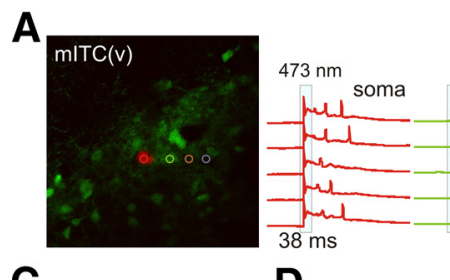

C
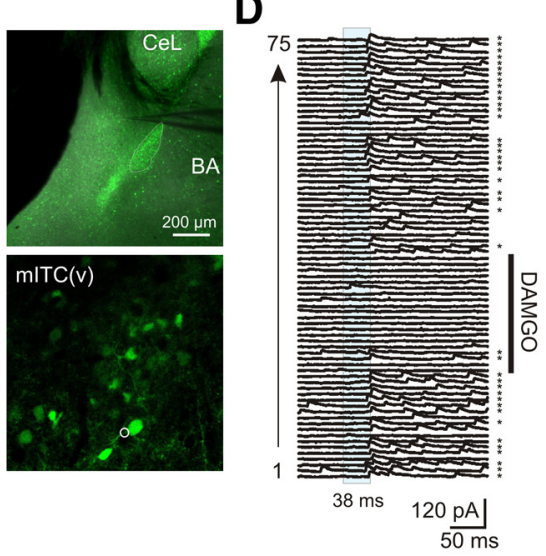

B

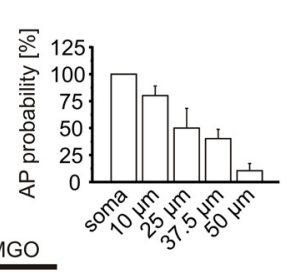

E

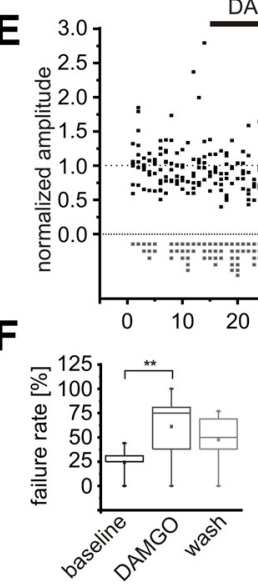

DAMGO

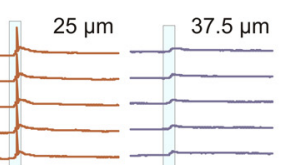
$\cdot$ $:$ an:

Figure 4. DAMGO decreases the synaptic reliability of GABAergic mITC-CeM synapses. A, Example of a current-clamp recording of $\mathrm{mITC}(\mathrm{v})$ neurons. The ROls for glutamate uncaging were set on the soma and at 10,25 , and $37.5 \mu \mathrm{m}$ distance from the soma of the recorded neuron. Scanning the ROIs on the soma, at 10 and $25 \mu \mathrm{m}$ distance, elicited AP firing in the recorded neuron but failed to evoke APs at $37.5 \mu \mathrm{m}$ distance. $\boldsymbol{B}$, Quantification of AP probability in $\mathrm{mITC}(\mathrm{v})$ neurons attributable to glutamate uncaging at various distances $(n=8 / 2)$. C, Presentation of a typical recording site in the CeM of a GAD67- eGFP mouse (top) and placement of the glutamate uncaging ROI within the mITC(v) cluster (bottom). D, GABA receptor-mediated responses in a CeM neuron recorded in the voltage-clamp mode at $-5 \mathrm{mV}$ (recorded sweep, 1 to 75 ; 15 s interstimulus interval). The elPS(s were evoked by stimulation within the mITC(v) cluster (C). Blue box represents the laser scan (38 ms duration). Asterisks represent responses counted as successes. Note the increased failure rate in the presence of DAMGO. E, Plot of all normalized success amplitudes (black squares) and failures (gray squares) recorded from nine CeM neurons. The black bar indicates DAMGO application $(n=9 / 7)$. $\boldsymbol{F}$, Quantification of the mean failure rate and success amplitudes of elPSCs in CeM neurons $(n=9 / 7)$.

increase of the normalized amplitudes during postTBS I in the presence of CTAP was significantly different from control (oneway ANOVA: $F_{(1,27)}=5.59, p=0.025$; Fig. $\left.5 D\right)$. Additionally, a comparison of time-point postTBS II during control and in the presence of CTAP revealed a significant difference (one-way ANOVA: $F_{(1,26)}=10.5, p=0.0033$; Fig. $\left.5 D\right)$. Concomitantly, the failure rate of eIPSCs increased in the control group after TBS (Fig. 5E). The failure rate was $22.5 \pm 4.1 \%$ during baseline stimulation in the control group $(n=13 / 8)$ and $17 \pm$ $2.7 \%$ in the presence of CTAP during baseline $(n=15 / 8)$. After TBS, in the control group, the mean failure rates increased to $150 \pm 45 \%$ normalized to baseline at time-point postTBS I and to $274.2 \pm 66.1 \%$ at time-point postTBS II (one-way ANOVA: $F_{(3,36)}=3.77, p=0.033$; post hoc test: baseline vs postTBS II, $p=0.021$; Fig. $5 E$ ). However, this significant increase of the normalized failure rate was not observed in the presence of CTAP. The normalized failure rate was reduced to $66.5 \pm 23 \%$ of baseline postTBS I and reversed to $125.3 \pm 55 \%$ of baseline postTBS II (Fig. 5E). These data indicate that MOR activation during TBS suppresses plasticity of the feedforward inhibition onto CeM neurons.

\section{Discussion}

While the IITC clusters within the amygdala have been shown to gate the input to the BLA complex (Marowsky et al., 2005), the mITCs mediate a modulation of the BLA output to the CeA (Likhtik et al., 2008; Amano et al., 2010; Palomares-Castillo et al., 2012). Moreover, mITCs receive additional input from the PFC and are involved in processes of fear extinction (Royer and Paré, 2002; Paré et al., 2003; Jüngling et al., 2008; Likhtik et al., 2008; Amano et al., 2010; Amir et al., 2011; Busti et al., 2011). ITCs in the amygdala are crucial modulators of local microcircuits. The high expression of MORs in these neurons indicates that endogenous MOR ligands have a major effect on neuronal processing within the amygdala. This might explain some of the effects of endogenous and exogenous MOR ligands on fear and pain processing. However, the cellular role of MORs in ITCs and consequent network effects remained elusive. Here, we provide evidence of the following: (1) mITCs functionally express MORs and are hyperpolarized during MOR stimulation; (2) an increase in membrane potassium conductance mediates the hyperpolarization; (3) a GABAergic feedforward inhibition mediated by the mITC-CeM connection is attenuated by MOR activation; whereas (4) BA excitatory inputs to mITCs or CeM neurons are not affected by MOR agonists.

The expression of MORs in mITCs has been described previously (Likhtik et al., 2008; Busti et al., 2011), and toxin-coupled MOR ligands have been used to eliminate these neurons before fear extinction retrieval in mice (Likhtik et al., 2008). Our findings regarding the DAMGO effects on the membrane potential are in line with previous findings that MORs act via $\mathrm{G}_{\mathrm{i} / \mathrm{o}}$-proteins (North et al., 1987) and increase potassium conductances via potassium inward rectifier channels. The persistence of the DAMGO-induced hyperpolarization or outward current in the presence of TTX, glutamate, and GABA receptor antagonists primarily excludes an indirect network effect via other transmitter systems. In addition, no DAMGO effects on the membrane potential were observed in BA PNs, even at higher concentrations. In agreement with previous observations, we detected DAMGO-induced outward currents in a subset of CeM neurons (Zhu and Pan, 2004).

On the network level, DAMGO decreased specifically the inhibitory component of disynaptic responses in CeM neurons after BA stimulation. It should be noted that electrical stimulation in the BA not only evokes glutamate release from BA PNs driving feedforward inhibition, but also remaining medial PFC fibers could be activated, additionally exciting mITCs (Cho et al., 2013). In fact, it has been shown previously that projections from the infralimbic cortex to mITCs are necessary for LTP at BLA-mITC synapses, increasing the excitation of mITCs and consequently the inhibition of CeM neurons, a process that is thought to underlie fear extinction (Amano et al., 2010). Although the exact origin of tested glutamatergic inputs has not been identified in the present study, it is interesting to note that a DAMGO-dependent suppression of the glutamatergic input was not detected in mITC(v) neu- 
A

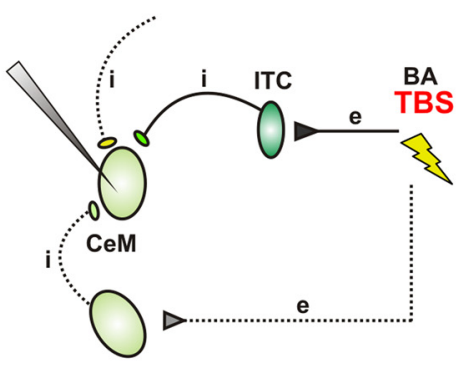

C

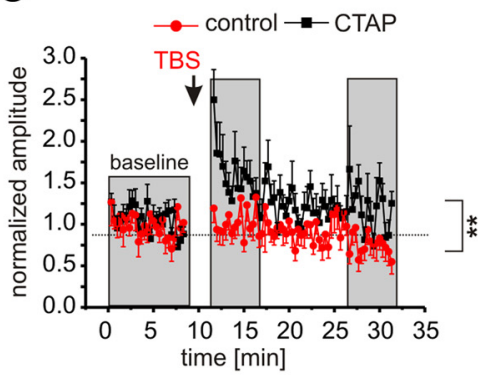

B
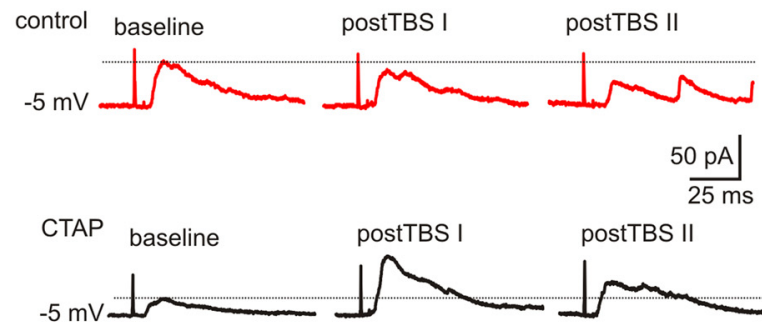

D

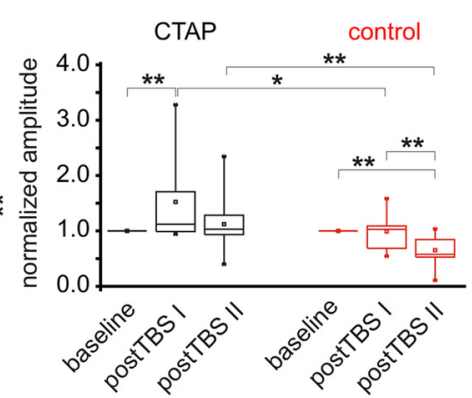

$\mathrm{E}$

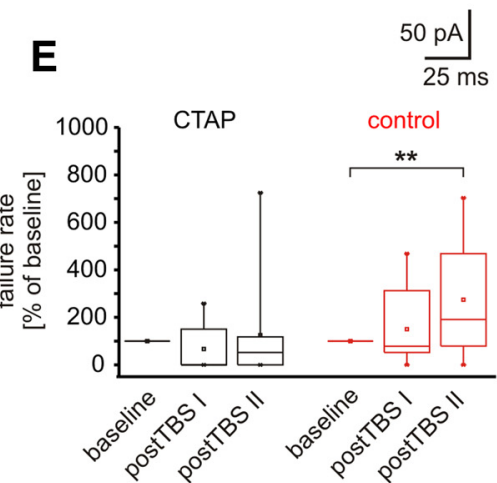

Figure 5. MOR antagonism allows plasticity of GABAergic feedforward inhibition in CeM neurons. $\boldsymbol{A}$, Scheme of putative synaptic connections contributing to feedforward inhibition of CeM neurons. TBS was performed in the BA, and CeM neurons were recorded in voltage-clamp mode at a holding potential of $-5 \mathrm{mV}$ to isolate GABAergic IPSCs. e, Excitatory; i, inhibitory. $\boldsymbol{B}$, Example recordings of CeM neurons during baseline conditions, postTBSI, and postTBS II. Recordings were performed either under control conditions (red) or in the presence of CTAP (black). C, Time course of the normalized amplitudes of evoked IPSCs in CeM neurons during control conditions ( $n=13 / 8$ ) and in the presence of CTAP ( $n=16 / 8$ ). Gray boxes represent time points used for analysis (baseline, postTBS I, and postTBS II). Mean amplitudes and failure rates were calculated from evoked responses within these time segments. $D$, $\boldsymbol{E}$, Quantification of the normalized success amplitudes $(\boldsymbol{D})$ and failure rates $(\boldsymbol{E})$ of elPSCs under control conditions $(n=13 / 8)$ and in the presence of CTAP $(n=15 / 8)$.

rons, indicating that the reduction of the feedforward inhibition onto CeM neurons must be located downstream of the excitatory input. In addition, using local glutamate uncaging, we provide evidence that a subset of $\operatorname{mITC}(\mathrm{v})$ neurons are involved in feedforward inhibition onto CeM neurons. Our data indicate that glutamate uncaging induces a spatially restricted excitation within the mITC(v) cluster, adding additional evidence to the existence of mITC-CeM synapses (Royer et al., 1999; Paré et al., 2003; Likhtik et al., 2008). The MOR effects on inhibitory, but not on excitatory, inputs in the $\mathrm{CeM}$ are in line with previously published data (Finnegan et al., 2005). Finnegan et al. (2005) proposed that the reduction of inhibition on CeM neurons is attributable to a presynaptic mechanism because the frequency of spontaneous IPSCs was strongly affected. Our data indicate that this reduction is in part mediated by MOR-dependent deactivation of mITCs. However, a contribution of GABAergic CeL or local CeM connections to the observed effect cannot be ruled out and demands additional investigation, because the CeL-CeM connection has been shown to be relevant for fear extinction ( $\mathrm{Du}$ varci and Pare, 2014). The feedforward inhibition mediated by mITCs is considered to underlie fear extinction (Likhtik et al., 2008; Amano et al., 2010; Busti et al., 2011), placing the mITCs in the center of a hypothesized extinction circuit between LA/BA extinction neurons and the CeM as the fear-relevant output station of the amygdala (Ehrlich et al., 2009; Amir et al., 2011; Busti et al., 2011). Therefore, the disinhibition of CeM neurons by cessation of mITC activity could induce increased anxiety and/or impairment of fear extinction.

The $\mu$-opioid system modulates LTP and synaptic plasticity at various locations (Dacher and Nugent, 2011). Here we provide evidence that an inhibitory disynaptic component recorded in CeM neurons during BA stimulation is enhanced by
TBS when recorded in the presence of the MOR antagonist CTAP. Similar findings have been made in the hippocampus when recording dentate granule cells while stimulating the lateral perforant path (Xie and Lewis, 1995). In that study, enhancement of IPSCs after high-frequency stimulation was only observed in the presence of a $\delta$-opioid receptor antagonist. MOR agonists are often found to allow LTP at excitatory synapses because of inhibition of local interneurons (Bramham and Sarvey, 1996). This gives rise to a scheme in which opioids favor excitatory LTP as a result of disinhibition, whereas enhancement of feedforward inhibition is enabled in their absence. Although the site of the observed plasticity is not clear, it seems likely that the excitatory input onto mITCs is enhanced by TBS (Huang et al., 2014), and CTAP blocks the inactivation of mITCs, resulting in an increased mITC output on CeM neurons.

The attenuation of CeM inhibition attributable to the inactivation of mITCs or local intra-CeA connections by the $\mu$-opioid system adds novel aspects to the complex system of endogenous opioids. Local release of endogenous ligands, such as enkephalin or endomorphin, would be adequate to diminish extinction learning and/or retrieval of fear extinction, as does the lesion of mITCs by MOR-specific toxins (Likhtik et al., 2008). Moreover, chronic morphine treatment has been shown to impair extinction of cued fear responses but not contextual fear responses in rats (Gu et al., 2008). It is tempting to hypothesize that the morphine-dependent attenuation of fear extinction is at least in part mediated by MOR activation in mITCs and their consecutive inactivation. Our findings make the mITCs an interesting neuronal substrate for anxiety disorders during drug abuse and nociception involving intra-amygdalar circuits. 


\section{References}

Amano T, Unal CT, Paré D (2010) Synaptic correlates of fear extinction in the amygdala. Nat Neurosci 13:489-494. CrossRef Medline

Amir A, Amano T, Pare D (2011) Physiological identification and infralimbic responsiveness of rat intercalated amygdala neurons. J Neurophysiol 105:3054-3066. CrossRef Medline

Bodnar RJ (2013) Endogenous opiates and behavior: 2012. Peptides 50:55-95. CrossRef Medline

Bramham CR, Sarvey JM (1996) Endogenous activation of mu and delta-1 opioid receptors is required for long-term potentiation induction in the lateral perforant path: dependence on GABAergic inhibition. J Neurosci 16:8123-8131. Medline

Busti D, Geracitano R, Whittle N, Dalezios Y, Mańko M, Kaufmann W, Sátzler K, Singewald N, Capogna M, Ferraguti F (2011) Different fear states engage distinct networks within the intercalated cell clusters of the amygdala. J Neurosci 31:5131-5144. CrossRef Medline

Cabral A, Ruggiero RN, Nobre MJ, Brandão ML, Castilho VM (2009) GABA and opioid mechanisms of the central amygdala underlie the withdrawal-potentiated startle from acute morphine. Prog Neuropsychopharmacol Biol Psychiatry 33:334-344. CrossRef Medline

Cho JH, Deisseroth K, Bolshakov VY (2013) Synaptic encoding of fear extinction in mPFC-amygdala circuits. Neuron 80:1491-1507. CrossRef Medline

Dacher M, Nugent FS (2011) Opiates and plasticity. Neuropharmacology 61:1088-1096. CrossRef Medline

Duvarci S, Pare D (2014) Amygdala microcircuits controlling learned fear. Neuron 82:966-980. CrossRef Medline

Ehrlich I, Humeau Y, Grenier F, Ciocchi S, Herry C, Lüthi A (2009) Amygdala inhibitory circuits and the control of fear memory. Neuron 62:757-771. CrossRef Medline

Finnegan TF, Chen SR, Pan HL (2005) Effect of the $\mu$ opioid on excitatory and inhibitory synaptic inputs to periaqueductal gray-projecting neurons in the amygdala. J Pharmacol Exp Ther 312:441-448. Medline

Gu C, Li P, Hu B, Ouyang X, Fu J, Gao J, Song Z, Han L, Ma Y, Tian S, Hu X (2008) Chronic morphine selectively impairs cued fear extinction in rats: implications for anxiety disorders associated with opiate use. Neuropsychopharmacology 33:666-673. CrossRef Medline

Huang CC, Chen CC, Liang YC, Hsu KS (2014) Long-term potentiation at excitatory synaptic inputs to the intercalated cell masses of the amygdala. Int J Neuropsychopharmacol 17:1233-1242. CrossRef Medline

Jüngling K, Seidenbecher T, Sosulina L, Lesting J, Sangha S, Clark SD, Okamura N, Duangdao DM, Xu YL, Reinscheid RK, Pape HC (2008) Neuropeptide S-mediated control of fear expression and extinction: role of intercalated GABAergic neurons in the amygdala. Neuron 59:298-310. CrossRef Medline

Khirug S, Huttu K, Ludwig A, Smirnov S, Voipio J, Rivera C, Kaila K, Khiroug L (2005) Distinct properties of functional KCC2 expression in immature mouse hippocampal neurons in culture and in acute slices. Eur J Neurosci 21:899-904. CrossRef Medline

Lange MD, Doengi M, Lesting J, Pape HC, Jüngling K (2012) Heterosynaptic long-term potentiation at interneuron-principal neuron synapses in the amygdala requires nitric oxide signalling. J Physiol 590:131-143. CrossRef Medline

Lange MD, Jüngling K, Paulukat L, Vieler M, Gaburro S, Sosulina L, Blaesse P, Sreepathi HK, Ferraguti F, Pape HC (2014) Glutamic acid decarboxylase 65: a link between GABAergic synaptic plasticity in the lateral amygdala and conditioned fear generalization. Neuropsychopharmacology 39:2211-2220. CrossRef Medline
Law PY, Wong YH, Loh HH (2000) Molecular mechanisms and regulation of opioid receptor signaling. Annu Rev Pharmacol Toxicol 40:389-430. CrossRef Medline

Likhtik E, Popa D, Apergis-Schoute J, Fidacaro GA, Paré D (2008) Amygdala intercalated neurons are required for expression of fear extinction. Nature 454:642-645. CrossRef Medline

Lutz PE, Kieffer BL (2013) Opioid receptors: distinct roles in mood disorders. Trends Neurosci 36:195-206. CrossRef Medline

Mańko M, Geracitano R, Capogna M (2011) Functional connectivity of the main intercalated nucleus of the mouse amygdala. J Physiol 589:19111925. CrossRef Medline

Marowsky A, Yanagawa Y, Obata K, Vogt KE (2005) A specialized subclass of interneurons mediates dopaminergic facilitation of amygdala function. Neuron 48:1025-1037. CrossRef Medline

North RA, Williams JT, Surprenant A, Christie MJ (1987) Mu and delta receptors belong to a family of receptors that are coupled to potassium channels. Proc Natl Acad Sci U S A 84:5487-5491. CrossRef Medline

Palomares-Castillo E, Hernández-Pérez OR, Pérez-Carrera D, CrespoRamírez M, Fuxe K, Pérez de la Mora M (2012) The intercalated paracapsular islands as a module for integration of signals regulating anxiety in the amygdala. Brain Res 1476:211-234. CrossRef Medline

Pape HC, Pare D (2010) Plastic synaptic networks of the amygdala for the acquisition, expression, and extinction of conditioned fear. Physiol Rev 90:419-463. CrossRef Medline

Paré D, Royer S, Smith Y, Lang EJ (2003) Contextual inhibitory gating of impulse traffic in the intra-amygdaloid network. Ann N Y Acad Sci 985: 78-91. CrossRef Medline

Royer S, Paré D (2002) Bidirectional synaptic plasticity in intercalated amygdala neurons and the extinction of conditioned fear responses. Neuroscience 115:455-462. CrossRef Medline

Royer S, Martina M, Paré D (1999) An inhibitory interface gates impulse traffic between the input and output stations of the amygdala. J Neurosci 19:10575-10583. Medline

Sandkühler J, Lee J (2013) How to erase memory traces of pain and fear. Trends Neurosci 36:343-352. CrossRef Medline

Tamamaki N, Yanagawa Y, Tomioka R, Miyazaki J, Obata K, Kaneko T (2003) Green fluorescent protein expression and colocalization with calretinin, parvalbumin, and somatostatin in the GAD67-GFP knock-in mouse. J Comp Neurol 467:60-79. CrossRef Medline

Tershner SA, Helmstetter FJ (2000) Antinociception produced by mu opioid receptor activation in the amygdala is partly dependent on activation of mu opioid and neurotensin receptors in the ventral periaqueductal gray. Brain Res 865:17-26. CrossRef Medline

Xie CW, Lewis DV (1995) Endogenous opioids regulate long-term potentiation of synaptic inhibition in the dentate gyrus of rat hippocampus. J Neurosci 15:3788-3795. Medline

Zadina JE, Martin-Schild S, Gerall AA, Kastin AJ, Hackler L, Ge LJ, Zhang X (1999) Endomorphins: novel endogenous mu-opiate receptor agonists in regions of high mu-opiate receptor density. Ann N Y Acad Sci 897: 136-144. CrossRef Medline

Zhu W, Pan ZZ (2004) Synaptic properties and postsynaptic opioid effects in rat central amygdala neurons. Neuroscience 127:871-879. CrossRef Medline

Zubieta JK, Smith YR, Bueller JA, Xu Y, Kilbourn MR, Jewett DM, Meyer CR, Koeppe RA, Stohler CS (2001) Regional mu opioid receptor regulation of sensory and affective dimensions of pain. Science 293:311-315. CrossRef Medline 